\title{
TV/Series
}

6 | 2014

Écho et reprise dans les séries télévisées (III) : de la métafiction à la transmédialité

\section{Relectures, réécritures, réinventions : Charmed ou l'art du recyclage postmoderne}

\section{Alexis Pichard}

\section{(2) OpenEdition}

\section{Journals}

Édition électronique

URL : http://journals.openedition.org/tvseries/319

DOI : 10.4000/tvseries.319

ISSN : 2266-0909

Éditeur

GRIC - Groupe de recherche Identités et Cultures

Référence électronique

Alexis Pichard, «Relectures, réécritures, réinventions : Charmed ou l'art du recyclage postmoderne », TV/Series [En ligne], 6 | 2014, mis en ligne le 01 décembre 2014, consulté le 10 décembre 2020. URL http://journals.openedition.org/tvseries/319; DOI : https://doi.org/10.4000/tvseries.319

\section{(c) (i) (9)}

TV/Series est mis à disposition selon les termes de la licence Creative Commons Attribution - Pas d'Utilisation Commerciale - Pas de Modification 4.0 International. 


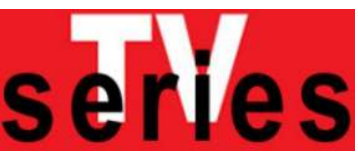

Relectures, réécritures, réinventions : Charmed ou l'art du recyclage postmoderne

Alexis PICHARD

Cet article se propose d'étudier l'esthétique postmoderne à travers la série fantastique Charmed. En nous reposant sur les travaux du théoricien français Gérard Genette, nous envisageons l'une des caractéristiques majeures des œuvres postmodernes : l'intertextualité, c'est-à-dire, au sens large, la relation référentielle qu'entretient un texte B avec un texte $\mathrm{A}$. Charmed est bien une série intertextuelle dans la mesure où elle recycle des œuvres passées et foisonne de références et de clins d'œil aux cultures populaire et élitaire. La série fait néanmoins un usage original de ces œuvres antérieures qui surgissent au fil des épisodes en les incorporant au sein de la diégèse et en leur conférant un rôle et un sens narratifs. La référence est réécrite pour amener un écho, une profondeur supplémentaire. $\mathrm{Au}$-delà de ce recyclage intertextuel, Charmed procède aussi de sa propre réécriture et mobilise une autre figure clé de l'esthétique postmoderne que Gérard Genette définit également: le palimpseste. Par son recours récurrent aux sauts dans le temps et aux fins alternatives, la série se présente comme une œuvre qui expérimente des trames narratives, se rature et se réécrit. En dernier lieu, nous nous intéressons au rapport ambigu que Charmed entretient avec l'influence, autrement dit les œuvres qui l'ont précédée. En partant du concept d'« anxiety of influence » théorisé par le critique américain Harold Bloom, qui joue sur l'idée que l'influence est une source d'angoisse mais aussi une source ardemment recherchée (polysémie du terme « anxiety » en anglais), nous montrons que la série propose une résolution dialectique à cette tension : elle semble arguer du fait que l'acceptation de l'origine permet à terme de faire naître l'originalité.

Hear now the words of the witches,

The secrets we hid in the night.

The oldest of gods are invoked here,

The great work of magic is sought.

In this night and in this hour,

I call upon the ancient powers.

Bring your power to we sisters three,

We want the power, give us the power.

Phoebe Halliwell ("Something Wicca This Way Comes," 1.01)

$\mathrm{C}^{\mathrm{s}}$

tte formule inaugurale prononcée par Phoebe Halliwell dans le premier épisode de Charmed (The WB, 1998-2006) place la question de la filiation et de l'influence au cœur de la série. La jeune femme invoque, sans le savoir, les pouvoirs ancestraux et, avec eux, tout un héritage intertextuel qui va donner cette saveur particulièrement ludique à Charmed. Le titre de la série lui-même suggère cette influence du passé: Charmed, soit "charmé », " ensorcelé » en français. Un sort a été jeté et l'on peut dès lors se demander qui en est la cible. S'agit-il des sœurs Halliwell que les prophéties nomment les « Charmed Ones », puissantes enchanteresses attendues de tout temps, ou bien des spectateurs que la série va ensorceler? À moins que l'objet du charme ne soit la série elle-même. 
La formule magique prononcée par Phoebe prend alors une dimension métafictionnelle car elle peut être entendue comme le manifeste de la série : à peine née, celle-ci affirme son identité. Le vers « in this night and in this hour » semble faire allusion au moment de la diffusion, impression renforcée par la présence de deux pronoms démonstratifs («this »), qui ancrent la diégèse dans une situation d'énonciation partagée, et par le fait qu'un épisode dure une heure. Cette identité sera façonnée par les dieux et pouvoirs anciens qui sont convoqués. Le couplet final est en cela intéressant puisqu'il démontre l'aspect revendiqué de cette exhortation. Les sœurs seront, en effet, investies de tout un héritage culturel, par un pouvoir intertextuel consenti qui va accompagner leurs aventures. Dès ses premiers instants, Charmed se présente comme la série de la reprise et du recyclage et s'ancre dans la mouvance postmoderne cathodique typique de son époque, aux côtés d'autres séries telles que Buffy the Vampire Slayer (The WB, 1997-2003) ou Xena, Warrior Princess (Syndication, 1995-2001). Ces séries datant des années 1990 sont caractérisées par leur usage détourné et amusé de la culture aussi bien populaire qu'élitaire, phénomène qui est constitutif de l'esthétique postmoderne ${ }^{1}$. Ainsi, pendant huit années, Charmed n'a eu de cesse de réinventer son héritage culturel, lequel lui a souvent servi de moteur narratif. L'intertextualité est cependant ici plus complexe et ne se limite pas au simple recyclage. En effet, la série propose une dynamique originale qui vise progressivement à se détacher de l'influence initiale pour façonner son propre héritage. Le recyclage postmoderne servirait alors à poser les fondations d'un nouveau cycle que Charmed tente de transmettre lors de son ultime épisode.

C'est précisément cette dynamique que nous nous proposons d'étudier au cours de cet article. Après avoir démontré que Charmed est une œuvre où foisonnent les références intertextuelles qui sont néanmoins recyclées de telle sorte qu'elles participent pleinement de la narration, nous analyserons la dimension palimpseste de la série, c'està-dire la manière dont elle se réécrit elle-même, rature ou efface certaines histoires ou bien certains personnages. Enfin, ces résurgences du passé intertextuel, de même que cette idée de palimpseste, trouvent pour symbole le manoir Halliwell qui officie comme le temple de toutes ces transformations. C'est un lieu hanté par le passé au sein duquel l'hypotexte - aussi bien le texte référence que la couche inférieure du palimpseste - prend vie, s'incarne, se répand et représente une menace que la série tente d'annihiler.

1 « Buffy: The Vampire Slayer, Xena: Warrior Princess, Dark Angel and Charmed (...) combined fantastic spectacle with a knowing recycling of plots and fragment from the media past. (...) Since the $1990 \mathrm{os}$, the intertextuality, recycling and self-referentiality of the postmodern form have also passed into the TV mainstream ", in Marc O'Day, "Postmodernism and Television ", in Stuart Sim (ed.), The Routledge Companion to Postmodernism, New York, Routledge, Second edition, 2005, p. 103-110. 


\section{Charmed, série hybride sous influence}

La série créée par Constance M. Burge se présente donc de prime abord comme un condensé ludique d'influences multiples. Films, livres, jeux vidéo, bandes dessinées, les sœurs Halliwell évoluent dans un maelström de références qu'elles réinventent au gré de leurs aventures magiques. Cette intertextualité est revendiquée dès le titre des épisodes. Substrat herméneutique de l'intrigue, le titre procède de la mise en relation d'une référence culturelle et d'un élément de la diégèse et est ainsi doublement référentiel. Ainsi, l'épisode «Womb Raider $^{2}$ » (4.21) pastiche le titre du jeu vidéo Tomb Raider qui suit les aventures de l'archéologue Lara Croft. Ce détournement drolatique tient au fait que, dans l'épisode, la diabolique Prophétesse tente l'impossible pour s'emparer du foetus démoniaque de Phoebe dans le but de régner sur le monde souterrain. La corrélation entre le titre et l'intrigue opère aussi dans "Save Private Leo » (4.17) qui fait référence à la fresque guerrière de Steven Spielberg, Save Private Ryan (1998). En effet, l'épisode relate le passé méconnu de Léo, l'ange gardien des sœurs Halliwell, qui servit d’infirmier et périt à Guadalcanal en 1942. Il est à présent traqué par ses anciens compagnons d'armes devenus fantômes en quête de vengeance. Le film de Spielberg se déroule également pendant la seconde guerre mondiale mais suit le débarquement de Normandie de 1944 et la recherche du soldat James Francis Ryan. Enfin, dernier exemple, «The Importance of Being Phoebe » (5.11) qui renvoie à la pièce The Importance of Being Earnest (1898) d'Oscar Wilde. L'épisode met en scène une démone avide de pouvoir qui va prendre l'apparence de Phoebe auprès de ses sœurs dans le but de les mener à leur perte. Cette duplicité identitaire n'est pas sans rappeler le jeu des dandys complices de la pièce de Wilde, Jack Worthing et Algernon Moncrieff, qui empruntent tous deux le nom d'Earnest (Constant en français) et s'inventent une vie parallèle afin d'échapper aux bienséances de la société victorienne3.

Si Charmed s'approprie de grandes références culturelles à un niveau préliminaire, elle présente également de nombreux recyclages formels. Ainsi, sa nature générique est-elle mise à l'épreuve et rendue hybride par les péripéties que traversent les sœurs Halliwell. Sur un ton toujours très amusé et décalé, la série va ainsi basculer d'un genre à l'autre, le plus souvent de manière inattendue. Dans l'épisode «The Good, the Bad and the Cursed » (3.14), dont le titre fait référence au célèbre western spaghetti The Good, the Bad and the Ugly (1966)

${ }^{2}$ Ce titre pourrait se traduire littéralement par «La pilleuse d'utérus » tandis que Tomb Raider signifie "La pilleuse de tombeaux ».

3 Pour l'analyse de l'intertextualité des titres de la série Charmed, nous renvoyons le lecteur à Alexis Pichard, Le Nouvel âge d'or des séries américaines, Paris, Le Manuscrit, 2011, p. 48-49. 
réalisé par Sergio Leone, la série revisite le western italien en déclinant toute l'esthétique du genre, des ralentis aux combats de saloons. Puis, dans les doubles épisodes "Oh My Goddess » (5.22-23) et «Valhalley of the Dolls »(6.1-2), la série revêt des allures de péplum. Phoebe, Piper et Paige sont successivement transformées, malgré elle, en déesses grecques pour venir à bout des Titans libérés des glaces, puis en Valkyries, ces divinités de la mythologie nordique qui recueillent et entraînent les âmes des meilleurs guerriers humains pour l'Ultime Combat. Dans ce dernier cas, il est amusant de remarquer que Charmed multiplie les clins d'œil en utilisant un extrait bien connu de «La Chevauchée des Valkyries » de Richard Wagner. Armures, épées, tridents, toges, gladiateurs, tous les clichés du genre sont tournés en dérision. Dans l'épisode « Charmed Noir » (7.8), Paige est piégée dans l'univers du film noir des années 1930. Devenue femme fatale, incontournable figure du genre, elle est confrontée à des gangsters en quête du convoité faucon birman dont le faucon maltais - allusion au roman de Dashiel Hammett et à l'adaptation filmique de John Huston - ne serait qu'une réplique. Entre fusillades, courses poursuites de voitures aux freins crissant et visites à des détectives véreux, la série s'amuse à nouveau avec les codes du genre, tout en les respectant à la lettre. Du point de vue de la technique, les particularités visuelles du film noir sont également rendues avec un souci d'authenticité puisque le réalisateur Michael Grossman multiplie les dutch angles (types de plans obliques), les contre-plongées et les projections d'ombres inquiétantes de gangsters sur les murs de sombres ruelles. En outre, l'épisode est en grande partie en noir et blanc, fait très rare à la télévision américaine contemporaine, et s'achève sur un "The End » dont la typographie réaffirme l'ambiance codifiée du genre.

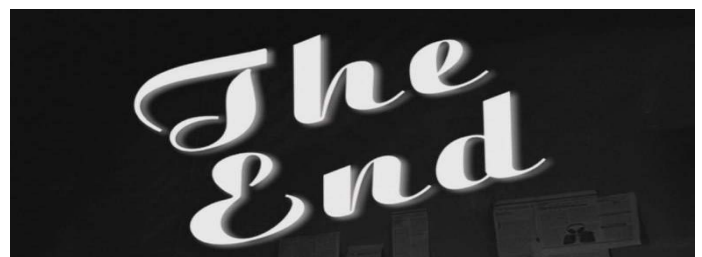

Fig. 1 : Plan final de l'épisode « Charmed Noir » (7.8)

Le recyclage effectué par la série s'effectue donc principalement sur le ton de l'hommage ou du pastiche. L'influence hypotextuelle affleure par moments pour être réinventée. Dans l'épisode " Primrose Empath » (3.8), Prue Halliwell libère un innocent d'une terrible malédiction : celui-ci est en effet capable de ressentir toutes les peines du monde qui l'entoure. Sans le savoir, Prue vient de venir en aide à un démon et se retrouve affublée de ce don qui va progressivement la 
conduire à la folie. Dans l'ultime affrontement qui l'oppose au démon Vincere, la sorcière parvient à maîtriser cette empathie destructrice et s'en sert alors comme une force. Le combat qui s'ensuit est un pastiche de The Matrix (Wachowski, 1999), film de science-fiction dont l'esthétique singulière est passée à la postérité. Charmed propose une relecture croisée de deux scènes de combat du film. En premier lieu, Prue s'élance dans les airs pour accabler Vincere d'une série de coups de pieds, mouvements similaires à l'attaque portée par Neo contre l'agent Smith lors de leur vigoureux corps à corps dans la station de métro. La fin du combat est, quant à elle, une relecture de la scène finale entre Neo et Smith. Prue prend alors l'avantage et se bat d'une seule main, son autre bras derrière le dos (en gros plan), avant de projeter son ennemi au sol d'un violent coup de poing. Cet enchaînement suit exactement celui de Neo, à l'exception du coup de pied qui devient coup de poing dans Charmed. Enfin, le coup de grâce porté par les deux héros se révèle identique. Neo et Prue s'élancent bras en avant et pénètrent le corps de leur opposant. Sous la pression interne, leur ennemi respectif convulse et se déforme avant de se disperser en mille morceaux dans une explosion d'un vert matriciel.

Si nous avons ici explicité et expliqué la nature et la dynamique de la relation intertextuelle, il semble important d'en interpréter la fonction au sein de la diégèse. En plus d'un hommage conscient, l'affleurement de l'hypotexte dans la série à un moment donné se révèle également porteur de sens. S'opère alors une corrélation entre la signification de la scène dans l'hypotexte et celle dans sa reprise hypertextuelle. La reprise est aussi bien stylistique que sémantique. Ainsi, la réécriture du combat final entre Neo et Smith joue le même rôle dans les deux récits. Il s'agit à chaque fois de l'acceptation et de la maîtrise d'un pouvoir démesuré. The Matrix suit précisément l'avènement de $\mathrm{Neo}$ et son parcours initiatique. La cinglante défaite de l'agent Smith démontre alors que l'ancien informaticien a embrassé sa destinée et qu'il sait se servir du pouvoir dont il a été investi. Il est l'Élu. C'est un cheminement semblable que l'on retrouve dans " Primrose Empath ", où Prue finit par transcender ses peurs et sa souffrance pour finalement accepter ses dons.

D'autres passages illustrent cette imbrication de l'esthétique et du sens. Dans l'épisode «The Torn Identity » (8.18), Coop - l'un des Cupidons - essaie tant bien que mal de réconcilier Phoebe avec l'amour. Tel un agent matrimonial, il pousse Michael, collègue de travail de Phoebe, à la courtiser. Hélas, celui-ci, franc et direct, s'avère récalcitrant aux exigences de son mentor. Néanmoins, convaincu que Michael est un parfait prétendant pour Phoebe, Coop promet de l'aider à lui faire la cour. Cela donne lieu à un dialogue qui pastiche la célèbre scène du balcon de Cyrano de Bergerac 4 (Rostand, 1897) dans laquelle

${ }_{4}^{4}$ III, 7. 
Cyrano aide son rival Christian à courtiser Roxane, dont les deux hommes sont épris. Incapable de parler d'amour, Christian accepte de répéter les mots doux, poétiques et passionnés que lui souffle Cyrano, dissimulé sous le balcon de Roxane. Charmed détourne cette configuration avec humour (au lieu de répéter «no, I'm not» il dit «no, I'm hot, » effectuant un saut de registre qui ne correspond pas au rôle qu'il doit tenir). Alors que Phoebe s'avance sur son balcon, elle est surprise de découvrir Michael en contrebas, les bras chargés de roses rouges. Comme chez Rostand, la belle se laisse séduire par la déclaration d'amour que le prétendant lui délivre, sans savoir que, tapi dans l'ombre du balcon, se cache le réel auteur de ces mots. À l'instar de Cyrano qui aime Roxane, Coop est éperdument épris de Phoebe. Une nouvelle fois, on observe que l'hypotexte n'est pas seulement l'objet d'une reprise ludique et amusée, mais qu'il a aussi une fonction narrative presque identique.

On peut également s'attarder sur l'épisode «Malice in Wonderland » (8.2) qui pastiche ostensiblement la série Sex and the City. À la différence des autres exemples mentionnés, la référence est annoncée dès le début de l'épisode. Les sœurs Halliwell, après de nombreuses péripéties, ont feint leur mort et ont embrassé une nouvelle existence sous de fausses identités. Le prologue de l'épisode met l'accent sur les difficultés qu'elles rencontrent dans cette quête d'une vie normale, chacune étant confrontée à des choix déterminants. Elles en viennent à évoquer la récente prémonition de Phoebe, laquelle s'est vue épouser Dex, collègue de travail qu'elle connaît à peine. Au cours de la discussion, Phoebe mentionne Carrie Bradshaw qu'elle va prendre comme modèle :

Piper. We were watching Sex and the City...

Phoebe. Speaking of which, did Carrie ever have to marry a man

that she did not know, huh?

Leo. Carrie?

Paige. Don't ask, they're on Sex and the City again.

Phoebe. No, the answer is no. Why can't we live our lives like they did?

Et alors que cette dernière s'empare du coffret DVD, Charmed s'imprègne soudain des codes et de l'ambiance de Sex and the City (voir figure 2). Phoebe devient la voix narrative de l'épisode, à la manière de Carrie dans Sex and the City. Charmed se pare alors de la dimension métaleptique de son aînée, autre procédé typiquement postmoderne qui « consiste en la transgression de la frontière entre deux niveaux narratifs en principe étanches, pour brouiller délibérément la frontière entre réalité et fiction 5 . " La métalepse est souvent l'apanage des séries à narration en voix-off qui tendent à

5 http://www.signosemio.com/genette/narratologie.asp, lien consulté le 11 décembre 2014. 
rompre le quatrième mur entre la fiction et le réel ${ }^{6}$. Le pastiche de Sex and the City est ici favorisé par la proximité des deux héroïnes, Carrie et Phoebe, qui tiennent chacune une chronique sur l'amour dans un journal. Par ailleurs, la musique de l'épisode reprend soigneusement les teintes jazzy de la série d'origine. Enfin, la dernière scène, qui s'achève sur un gros plan de l'écran d'ordinateur, rappelle un passage présent dans la plupart des épisodes de Sex and the City, durant lequel Carrie prononce sa célèbre réplique-ritournelle introductive: «I couldn't help but wonder... »

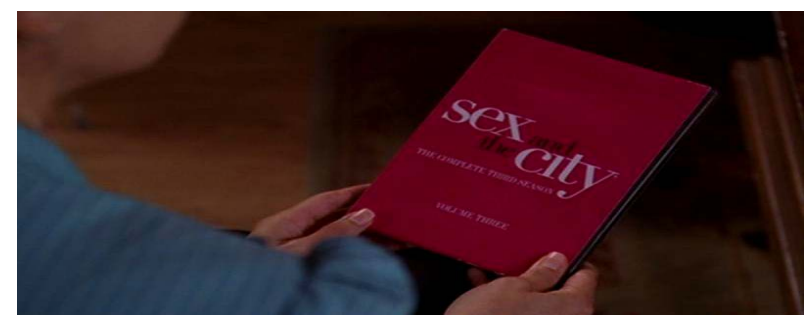

Fig. 2 : Sex and the City pénètre l'univers diégétique de Charmed (8.2)

Venant parachever cet épisode pastiche, la présence de l'acteur Jason Lewis offre un cas intéressant d'hypertextualité puisque celui-ci a également tenu un rôle régulier dans Sex and the City. Il y interprétait Smith Jerrod, un acteur en devenir propulsé dans le show bizness par Samantha. Dans Charmed, il joue Dex Lawson, prétendant et mari - le temps de quelques heures - de Phoebe. Si le passage d'un acteur d'une série à une autre est un phénomène fréquent, il est néanmoins plus rare d'employer un acteur au sein d'une série qui pastiche celle dans laquelle il tenait auparavant un rôle semblable. Plus qu'un acteur, on a le sentiment que c'est un personnage de Sex and the City qui est transféré dans Charmed. Ainsi, la présence de Lewis dans les deux séries semble aller plus loin que l'acception traditionnelle de l'hypertextualité définie par Gérard Genette. Il ne s'agit plus d'une simple transformation d'un univers fictionnel par un autre mais bien d'une transgression de l'un dans l'autre. L'univers de Sex and the City pénètre la diégèse de Charmed et l'on observe ainsi une coprésence des deux séries. Cela est rendu d'autant plus patent que le prénom du personnage joué par Jason Lewis est Dex, qui s'entend presque comme le « sex » de Sex and the City. Cette assonance est d'ailleurs soulignée par un jeu réflexif de Piper :

Last year you had a premonition that you would have a daughter which means that this year you have to have a little sex and get pregnant. And since Dex, which coincidentally rhymes

${ }^{6}$ Que l'on songe à Meredith dans Grey's Anatomy (ABC, 2004- ) ou à Mary Alice dans Desperate Housewives (ABC, 2004-2012). 
with sex, could be the father! (nous soulignons)

Cet épisode représente donc un cas peu fréquent qui mêle hypertextualité et intertextualité : alors que Charmed pastiche minutieusement Sex and the City, ce qui tient de l'hypertextualité, la série donne aussi à voir des espaces diégétiques aux frontières ténues, espaces qui se fondent sans distinction les uns dans les autres, ce qui illustre une définition plus étroite de l'intertextualité. L'esthétique postmoderne est à son comble.

L'influence s'exerce également à travers la citation qui se révèle aussi porteuse de sens. Dans l'épisode « The Seven Year Witch » (7.16) qui voit le retour momentané de Cole Turner, grand amour maléfique de Phoebe, Piper tombe dans le coma après avoir été infectée par un poison mortel. Dans les limbes, elle rencontre l'esprit errant de Cole. Voyant passer Phoebe devant lui, celui-ci cite un vers de Romeo and Juliet : « O [beauty], she doth teach the torches to burn bright !7 » Ces quelques mots sont prononcés par Romeo alors qu'il vient d'apercevoir Juliet chez les Capulet. Interloqué par la beauté de la jeune femme, il exprime son admiration avant d'être découvert par Tybalt, neveu de lady Capulet. C'est la première rencontre entre les deux amants maudits $(I, 5)$. Charmed intègre la citation dans un contexte semblable. Cole prononce ce vers alors que Phoebe ne peut le voir, ce qui est aussi le cas de Romeo. Étrangement, au moment où Cole déclame ce vers, Phoebe s'arrête brusquement et recule pour regarder dans sa direction, croyant avoir entendu quelque chose. Mais elle ne voit rien. La citation traverserait les différents mondes comme elle traverse les âges. Cependant, alors que Romeo prononce sa tirade enflammée («the torches to burn bright ») au premier regard porté sur Juliet, présageant la relation passionnée des deux amants, Cole se limite au premier vers, deux ans après avoir été vaincu par Phoebe ${ }^{8}$. On entend ici l'ironie et l'amertume de l'utilisation de ce vers qui ne suggère pas l'idylle à venir mais bien la fin de l'histoire des deux amants. Il faut rappeler que le couple formé par Cole et Phoebe était déjà très inspiré par Romeo et Juliet, tous deux appartenant à des camps antagonistes, le bien et le mal. Ainsi, l'intertexte shakespearien convoque la connaissance de la pièce et celle de l'histoire de la série9.

${ }_{7}$ Romeo and Juliet (1597), I, 5. Notons que la citation est signalée par la réplique de Piper : "Since when do you quote Shakespeare?"

8 «Centenial Charmed » $(5.12)$.

9 On pourra relever d'autres références à l'univers shakespearien, notamment à MacBeth (1606). Ainsi, la présence dans Charmed d'un trio de sorcières n'est pas sans rappeler celui de la pièce de Shakespeare. Cet écho est d'autant plus marquant que le titre du premier épisode de la série emprunte à une réplique de MacBeth prononcée par l'une des sorcières : « By the pricking of my thumbs, Something wicked this way comes. " Charmed reprend la dernière partie de cette réplique et la détourne, comme à son habitude, en "Something wicca this way comes". On voit ainsi s'établir un dialogue entre les sorcière shakespeariennes ("weird sisters ») et celles de Charmed, nettement moins effrayantes. Il 
Charmed se présente donc comme une série dans laquelle on retrouve les traces plus ou moins subtiles d'autres œuvres artistiques. Ces jeux de reprises et d'échos donnent lieu à d'étonnantes transformations, telles que le pastiche ou bien la réécriture, et illustrent ainsi l'aspect métafictionnel et postmoderne de la série. On peut d'ailleurs remarquer que Charmed ne se limite pas seulement à une réécriture intertextuelle puisqu'elle procède également de sa propre réécriture, ce qui lui permet de mettre doublement l'accent sur son caractère fictionnel.

\section{Réécrire la diégèse : entre alternative et altération}

La réécriture, processus essentiel pour insérer la référence dans un nouveau cycle, celui de la série, s'opère également au niveau de la diégèse. On ne cherche alors plus à réécrire et à réévaluer l'héritage intertextuel mais bien l'histoire proposée dans la série. Ces phénomènes émaillent les huit saisons de Charmed et donnent à voir une série palimpseste. Gérard Genette définit le palimpseste comme « tout texte dérivé d'un texte antérieur, par transformation [...] ou par [...] imitation ${ }^{10}$. » Il s'agit plus généralement d'une œuvre d'art dans laquelle on perçoit en filigrane les traces d'une autre œuvre d'art. On s'appuiera ici, d'une part, sur les voyages dans le temps qui sont légion au sein de la série et, d'autre part, sur les fins alternatives proposées par certains épisodes.

Dans Charmed, le saut dans le temps repose toujours sur l'idée que le passé doit être altéré afin de réécrire le présent et ainsi sauver la lignée des sœurs Halliwell sans cesse menacée. Dans l'épisode « That 70s show» (1.17), les sœurs doivent retourner dans les années 1970 pour empêcher leur mère Patty de conclure un pacte maléfique avec un démon qui l'immunisera face au pouvoir des trois. Dans "All Halliwell's Eve » (3.4), elles sont envoyées dans l'Amérique des Pères Fondateurs afin de permettre la naissance de Melinda Warren, fondatrice de la lignée Halliwell. Enfin, l'intrigue de la sixième saison repose entièrement sur ce procédé en inversant la perspective. Cette fois, c'est Chris, jeune homme âgé d'une vingtaine d'années, qui surgit du futur pour prévenir les sœurs Halliwell qu'un danger plane sur l'avenir de Wyatt, fils de Piper. L'intrigue principale tournera autour de ce mystère : quelle est cette menace ? Et comment l'arrêter ? Nous apprendrons à mi-parcours que Chris est en fait le frère cadet de Wyatt revenu pour empêcher celui-ci de basculer vers la magie noire. Ainsi,

n'est d'ailleurs pas anodin que le mot «wicked » ait été remplacé par «wicca » : à la cruauté et à la laideur promise, se substituent la magie et le charme enchanteur. Le tragique de la pièce est d'emblée déjoué dans la série.

${ }^{10}$ Gérard Genette, Palimpsestes : la littérature au second degré, Paris, Éditions du Seuil, 1982. 
par ses distorsions et manipulations temporelles, Charmed semble se rapprocher des récits non-linéaires qui sont l'apanage du roman postmoderne.

Au-delà de ces altérations narratives, la série propose aussi un autre type de réécriture: les fins alternatives. On retrouve ce mécanisme constitutif du postmodernisme littéraire dans de nombreuses œuvres, comme dans The French Lieutenant's Woman (1969) de John Fowles qui nous propose pas moins de trois fins possibles à son récit sans en privilégier aucune. Dans Charmed, la portée de ce procédé est limitée par la nature sérielle du programme qui impose que la dernière fin offerte dans un épisode apporte la conclusion de l'intrigue. Nul doute ne doit subsister, l'épisode doit être bouclé de manière à ce que le spectateur sache où la série recommencera.

Le couple d'épisodes formé par "Déjà Vu All Over Again" (1.22) et "All Hell Breaks Loose » (3.22) donne à voir deux processus de réécriture enchâssés, qui semblent se faire écho. Ces deux season finales reposent sur la même idée d'un temps circulaire, d'une journée qui se répète à l'infini. On retrouve le même démon, Tempus (David Carradine), responsable de la boucle temporelle dans les deux cas, que l'on découvre dans "Déjà $\mathrm{Vu}$ All Over Again » et qui est à nouveau mentionné dans « All Hell Breaks Loose ». Ce couple d'épisodes forme ainsi un doublon qui repose sur une série de répétitions et d'échos comportant malgré tout quelques différences très sensibles. Si la journée dans le final de la saison 1 se répète deux fois, celle du final de la saison 3 ne se répète qu'une fois. Autre écho intéressant, les sœurs, au cours d'un combat semblable, sont projetées à travers différentes pièces du manoir. Dans le premier cas, Piper traverse une porte vitrée donnant sur le solarium. Dans le second cas, Prue et Piper traversent le mur situé juste à côté de cette même porte. Une nouvelle fois la scène semble identique alors qu'elle est subrepticement déformée. On mentionnera également que, dans les deux épisodes, un personnage important perd la vie : d'abord Andy Trudeau, l'ami des sœurs, puis Prue. Enfin, tandis que le dernier plan montre les portes du manoir Halliwell se refermer, dans le premier cas, c'est Prue qui les referme par magie et, dans le second cas, c'est le démon Shax qui, ayant accompli sa mission, se replie dans une tornade dont la violence va jusqu'à briser les vitres teintées des portes. S'opère donc ici un recyclage interne qui donne lieu à une réécriture de la diégèse : le même en différent. Sans oublier que l'histoire des deux épisodes se fonde sur le principe de réécriture puisque la journée est relancée pour que son issue soit différente.

Par ailleurs, l'histoire familiale des Halliwell a aussi été sujette à des altérations ayant trait à la production même de la série. Le père des sœurs, Victor Bennett, apparaît pour la première fois dans l'épisode «Thank you for not morphing» (1.03) sous les traits d'Anthony 
Denison. L'acteur n'ayant pas souhaité reprendre son rôle, Victor Bennett est ensuite interprété par James Read. La série, tel un palimpseste, est ainsi émaillée de ratures et d'effacements. L'exemple le plus probant est sans doute la résurrection du pouvoir des trois dans l'épisode « Charmed Again » (4.01-02). À l'issue de la troisième saison, le départ de Shannen Doherty ${ }^{11}$, interprète de Prue Halliwell, marque la fin du premier pouvoir des trois. Les dernières secondes de l'épilogue apocalyptique nous montrent la victoire de la Source et de son assassin Shax, lequel est parvenu à tuer Piper et Prue, laissant le téléspectateur désemparé face à ce cliffhanger inédit. " Charmed Again » constitue le renouveau de la série et du pouvoir des trois, comme l'indique la présence de l'adverbe « again ». Pour la première fois dans l'histoire de la série, le titre d'un épisode contient le mot " charmed » (ce ne sera pas la dernière). Ce procédé sert à réaffirmer l'identité de la série après une période de doute et à amorcer un nouveau cycle. Le sort va de nouveau opérer, le pouvoir des trois va être ressuscité, la série va se réinventer. Cet épisode charnière sonne comme un nouveau départ, marqué par l'inhumation de Prue dès les premières minutes ${ }^{12}$, et pourrait signifier l'enterrement symbolique des trois premières années de la série. Pourtant, le titre de l'épisode semble d'abord annoncer que la série restera la même, que Charmed sera encore Charmed. Cette promesse apparaît dans toute son ambiguïté dans les premières secondes de l'épisode lorsque Piper, assise dans le grenier, essaie désespérément plusieurs formules magiques afin de faire revenir Prue. En effet, une voix se fait entendre dans le couloir. Piper, filmée en plan rapproché poitrine, relève la tête et prononce le nom de sa défunte sœur avec un soudain regain d'espoir. À ce moment précis, le spectateur rejoint ce sentiment et veut aussi croire au retour de Prue. Le contre-champ qui suit nous montre l'entrée du grenier, vide. Prue est bien morte, elle ne reviendra pas, comme l'annonce Phoebe au cours d'une réplique qui semble aussi bien destinée à sa sœur qu'au spectateur : "We have tried every magical way to bring her back. But we can't. She's gone. »

Le titre de l'épisode apparaît dès lors comme une tromperie. Charmed ne sera plus jamais Charmed. Pour autant, comment continuer à justifier un tel titre ? La dynamique de la série reposant sur

${ }^{11}$ Ce départ inattendu fut très médiatisé en mai 2001 mais les raisons en restent néanmoins assez obscures. Beaucoup de journalistes ont expliqué la décision de Shannen Doherty par les différends artistiques qu'elle rencontrait avec la production, qui souhaitait donner une orientation plus sombre à la série afin de l'éloigner d'un public trop féminin. Officieusement, ce serait la rivalité avec sa partenaire, Alyssa Milano, qui aurait poussé la production à se séparer de Doherty. Ce départ fracassant n'était alors pas le premier de l'actrice puisque, quelques années plus tôt, elle quittait brusquement Beverly Hills suite à une mésentente avec la production. Ou quand le recyclage dépasse le cadre de la diégèse...

${ }^{12}$ Cette scène d'enterrement inaugurale semble d'ailleurs annoncer l'épisode "Still Charmed and Kicking » (8.1) au début duquel se déroule une veillée funèbre en l'honneur des sœurs Halliwell, mortes aux yeux du monde entier. 
un trio de sorcières, les scénaristes vont créer une nouvelle sœur pour reformer le pouvoir des trois. Au personnage de Prue succèdera Paige. À travers ce prénom, la série affirme son désir de renouveau, renouveau ambivalent puisqu'il repose sur un recyclage. Notons d'abord que la phonie du prénom "Paige » est identique à celle de «page » en anglais. Cette quatrième sœur, dont l'existence nous est révélée et expliquée au cours de l'épisode, est donc intrinsèquement liée à l'écriture et à la page vierge qui reste à écrire. Cette relation est mise en exergue par l'épisode « A Paige from the Past » (4.10), titre qui substitue à la page le nom de Paige. Néanmoins, cette page vierge ne l'est peut-être qu'en apparence puisque Paige semble être une nouvelle version, certes différente à certains égards, de Prue. En effet, comme elle, son prénom commence par un « $\mathrm{P}$ ». Toutes deux sont brunes, peuvent déplacer les objets par la pensée et se transporter dans un espace différent. Autrement dit, Paige serait un personnage palimpseste derrière lequel on retrouve l'influence de Prue que l'on devine en transparence.

Ainsi, les métamorphoses physiques au sein de la famille Halliwell illustrent un cas de réécriture diégétique qui voit un personnage ${ }^{13}$ interprété par différents acteurs. À cela, nous pouvons ajouter deux types de réécritures "métamorphiques". Comme l'indique l'étymologie du terme en grec ancien, nous sommes à présent " au-delà » (méta) de la «forme » (morphê). La transformation métamorphique se comprendrait alors comme un changement aussi bien physique qu'identitaire, formel et structurel. On distingue, d'une part, les cas où un acteur incarne différents personnages et, d'autre part, ceux où un personnage incarne un autre personnage.

Connue pour son rôle de rédactrice en chef du Bay Mirror, journal où travaille Phoebe, Rebecca Balding a d'abord fait une apparition dans la première saison ${ }^{14}$ de Charmed sous les traits d'un autre personnage, celui de Jackie, tante d'Aviva, jeune sorcière sous l'influence d'une démone. La même actrice incarne donc deux personnages différents, situation que la série oblitère complètement. On peut aussi voir dans ce procédé un nouvel hommage au théâtre de Shakespeare qui, tel qu'il était joué au XVII ${ }^{\mathrm{e}}$ siècle, était marqué par le fait que les acteurs jouaient souvent plusieurs rôles au sein d'une même pièce, ce qui rendait prépondérante la question du masque. Imitant le théâtre élisabéthain, Charmed va recourir au masque dans le cas de l'acteur Michael Bailey Smith, qui incarne à lui seul quatre démons réguliers de la série. L'aspect «métamorphique» de la transformation est rendu évident du fait que chaque démon se démarque par un physique idiosyncratique, de Shax, monstre imposant

${ }^{13}$ Considérons ici Prue et Paige comme un seul et même personnage car elles ont toutes deux un rôle similaire au sein de la famille et de la série.

14 « The Fourth Sister » (1.7). 
au corps bleu, à Belthazor, à la peau rouge marquée de volutes noires, en passant par les Grimlocks, démons souterrains à la peau blanche et aux yeux cerclés de rouge sang. L'acteur est ainsi devenu cette roche altérée par transformation métamorphique (au sens scientifique de ce terme), ici générées par l'univers fictionnel. Ainsi, par ce recyclage conscient des acteurs, lesquels sont amenés à jouer plusieurs rôles, Charmed réaffirme son caractère métafictionnel en revendiquant précisément son statut une fiction. Le cas précité de Belthazor illustre bien ce point, de même qu'il montre la mise en abyme métafictionnelle à l'œuvre dans la série puisque le maquillage du démon est inspiré de celui de Darth Maul, Sith malfaisant de Star Wars: The Phantom Menace (Lucas, 1999). Autrement dit, on recycle consciemment un acteur pour un autre rôle au sein du même programme mais, afin de le masquer, on le grime en reprenant les traits d'un personnage d'une autre fiction.

À ces métamorphoses diégétiques s'ajoutent des métamorphoses intradiégétiques, qui voient un personnage incarner un autre personnage. La série en propose un nombre considérable tant la magie invite au déguisement, aux apparences et à la tromperie, se rapprochant une nouvelle fois du théâtre shakespearien dont ces pratiques étaient constitutives ${ }^{15}$. Les sœurs Halliwell sont les premières victimes de ces transformations, en particulier Phoebe, qui aura tour à tour été une Banshee, démone aux allures de vampire, une ménagère tout droit sortie des années cinquante, une sirène, une super héroïne digne des comics américains ou bien encore Cendrillon. La récurrence de ces métamorphoses la conduit d'ailleurs à de savoureux constats ironiques, notamment lorsque, devenue génie, elle s'exclame : "And why do I always get stuck with the wig' ${ }^{16}$ ? " Une nouvelle fois, ce commentaire se pare d'une résonnance métafictionnelle, ne sachant plus très bien si le locuteur est Phoebe ou son interprète, Alyssa Milano. Ces mises en abyme fictionnelles sont également le fondement de nombreux épisodes reposant sur l'usurpation d'identité, le plus souvent à des fins machiavéliques, dans lesquels l'identité et l'histoire des sœurs sont réécrites.

${ }_{15}$ On peut penser au roi Polixenes qui se déguise en berger dans The Winter's Tale pour empêcher le mariage de son fils avec une bergère.

16 «I Dream of Phoebe » (6.15). 


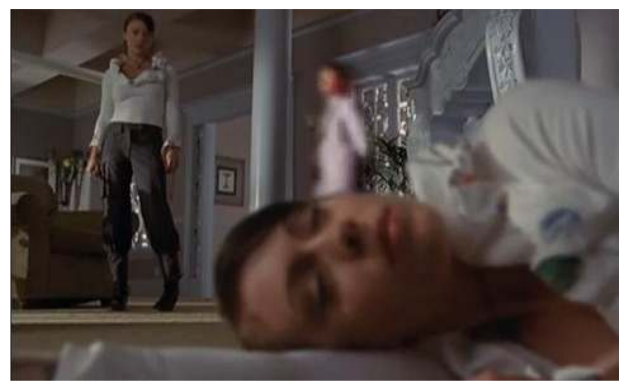

Fig. 3 : Phoebe et son double démoniaque (5.11)

C'est notamment l'objet de « The Importance of Being Phoebe » qui voit la démone Kaira prendre l'apparence de Phoebe (voir figure 3), mais aussi de "The Power of Three Blondes » (6.04) où les sœurs Halliwell sont remplacées par un trio de sœurs démoniaques. Plus tard, ce motif narratif est une nouvelle fois décliné dans " Repo Manor » (8.13) au cours duquel trois sœurs démones prennent l'identité des sœurs Halliwell pour s'emparer de leurs pouvoirs dans le but de mettre à mal le système esclavagiste qui régit le monde souterrain. On voit donc que chacune de ces transformations métamorphiques conjugue changement physique et identitaire. Les personnages réinterprétés voient leur génome réécrit, modifié, pérennisant l'image d'une série au caractère instable où les identités sont volatiles.

Ces exemples mettent ainsi en évidence l'aspect palimpseste de Charmed, laquelle ne recycle pas seulement les textes qui l'ont précédée mais aussi son propre texte. En donnant à voir les expérimentations auxquelles elle se livre, les effacements et les altérations qu'elle opère sur sa propre diégèse, la série revendique une nouvelle fois son caractère fictif et s'engage à nouveau dans un jeu ironique et métafictionnel typique de l'esthétique postmoderne. Finalement, si l'on essaie de résumer l'ensemble des analyses faites à propos du recyclage intertextuel et de l'idée de palimpseste, on peut théoriser une certaine poétique de l'influence à l'œuvre dans Charmed. En effet, la série serait travaillée par une tension entre désir d'imitation et désir d'originalité, à laquelle elle proposerait une résolution dialectique. Afin d'explorer ce cheminement, nous nous proposons d'examiner ici ce lieu central et hautement symbolique de la série : le manoir Halliwell.

\section{Le Manoir Halliwell : temple palimpseste}

Nous avons démontré jusqu'ici que Charmed est une série hybride, sous influences multiples, au sein de laquelle s'enchâssent différentes réécritures. Dans cette dernière partie, nous nous 
attarderons plus particulièrement sur le lieu où se réalisent la plupart des mécanismes intertextuels, le manoir Halliwell. Il symbolise à lui seul la notion d'héritage présente au cœur de la série. L'étymologie même du terme " manoir » nous invite à cette interprétation puisqu'il provient du verbe latin manere, autrement dit "demeurer", « habiter ». Ce qui demeure, dans un premier temps, c'est la magie de la lignée Halliwell depuis Mélinda Warren jusqu'à Chris Halliwell (deuxième fils de Piper né à la fin de la sixième saison). Cette filiation est incarnée par le Livre des Ombres, grimoire transmis et complété de génération en génération, véritable encyclopédie sur le monde de la magie. Il comporte à lui seul toute la mythologie propre à Charmed, établit un dialogue entre passé et présent et agit ainsi comme le métonyme de l'intertextualité. Par ailleurs, le manoir est aussi le garant de la pérennité de l'influence. De manière récurrente, il déclenche l'intertextualité. Il exerce une influence ambiguë tant l'hypotexte conjuré peut être salvateur, comme nous l'avons vu dans l'épisode «Primrose Empath » où Prue est investie des pouvoirs de Neo, héros de la séries des films The Matrix (1998-2003), ou bien, au contraire, mortifère. On peut dès lors voir affleurer une "angoisse de l'influence » («anxiety of influence»), concept énoncé par Harold Bloom $^{17}$ à propos de la poésie. Le critique anglais argue que la créativité d'un poète est naturellement inspirée par les poètes qui l'ont précédé. Cette réflexion montre le caractère ambivalent de cette relation qui inspire et nuit dans le même temps à l'originalité. En effet, cette angoisse de l'influence réside dans l'impossibilité supposée de produire un texte neuf et authentique qui soit libéré de toute influence. Selon Bloom, tout texte est tributaire de ceux qui l'ont précédé. Appliqué à Charmed, ce concept permet d'entrevoir la tension existante entre une influence fondatrice qui perdure jusqu'à nuire in fine et un désir d'originalité. Le manoir apparaît alors comme l'épicentre de ce conflit interne et joue un rôle particulièrement ambigu.

L'histoire même du lieu a longtemps fait la part belle à la légende. En effet, durant des années, on raconta que le manoir Halliwell était précisément celui qui avait été bâti à la fin des années 1950 pour les besoins du film Psycho d'Alfred Hitchcock. Dès le début, le manoir apparaissait donc habité par une influence inquiétante et dévoilait sa nature palimpsestueuse. Néanmoins, malgré d'indéniables similitudes, les bâtisses présentes dans les deux œuvres s'avèrent différentes et ne partagent pas la même histoire. Le légendaire manoir de Psycho, construit dans les studios d'Universal, fut détruit à la fin des années 1970 puis reconstruit en 1981 pour le tournage de Psycho 2 de Richard Franklin. Le manoir Halliwell, quant à lui, a été bâti à la fin du

17 Harold Bloom, The Anxiety of Influence: A Theory of Poetics, New York, Oxford University Press, 1997. 
$\mathrm{XIX}^{\mathrm{e}}$ siècle sur les hauteurs en bordure de Los Angeles et il est encore habité. Malgré tout, la filiation légendaire est exploitée dans Charmed, comme si le manoir victorien était toujours hanté par Norman Bates. Le passé se fait des plus néfastes. On mentionnera, par exemple, le Nexus, source de pouvoir instable qui jaillit des entrailles de la cave à plusieurs reprises au cours de la série et opère une influence des plus funestes. Dans l'épisode "Is there a Woogie in the House? » (1.15), le Nexus prend possession du corps de Phoebe et, par là-même, du manoir. L'hypotexte, partie inférieure du palimpseste, semble reprendre ses droits et combattre l'histoire nouvelle qui s'écrit. Cela est particulièrement remarquable dans l'épisode où Prue tente de rentrer dans le manoir et qu'elle en est alors rejetée violemment, mais aussi, de manière plus métaphorique, quand le papier peint aux murs se décolle, ce qui symbolise bien les couches constitutives du palimpseste. Néanmoins, cette possession mortifère est finalement mise en échec par les sœurs et le Nexus - sorte de dense fumée noire - est aspirée par la faille dont il s'était échappé plus tôt.

Sur un ton plus drolatique, l'influence hichcockienne originelle surgit aussi lors dans l'épisode "Chick Flick» (2.18) où des personnages de films d'horreur traversent l'écran de cinéma et assaillent le manoir. Poursuivie par l'un d'entre eux, Piper se réfugie dans la douche avant de s'apercevoir de son erreur: " I am being stalked by psycho killers and I hide in the shower!" L'occurrence du mot «psycho" réaffirme l'intertexte, de même que la douche, métonyme mémorable du film d'Hichcock (voir figure 4). Mais l'influence est à ce moment-là déjouée et Piper ne subira pas le même sort que Marion Crane. En effet, l'ombre menaçante qui se dessine sur le rideau de douche n'est autre que celle de sa sœur, Prue.

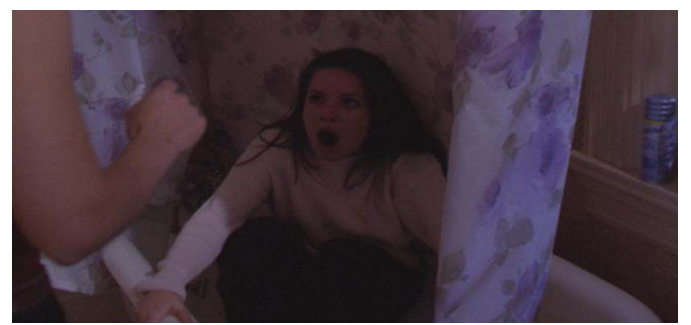

Fig. 4 : Charmed pastiche le film Psycho (2.18)

Cette influence du passé est aussi visible dans les résurgences diégétiques et extradiégétiques que le manoir provoque. Qu'il s'agisse de vies ou de carrières antérieures, le manoir ancre la série dans le passé à différents niveaux narratifs. L'épisode "Pardon My Past» (2.14) s'ouvre sur une scène tout à fait anodine. Piper et Prue organisent une soirée avec des amis. Phoebe, en pleines révisions pour 
ses examens, descend signaler à ses sœurs qu'elle a besoin de silence. La fête prend doucement fin, les invités s'en vont. C'est alors que Phoebe entend une musique jazzy et une ambiance festive dans le salon. Courroucée par ce vacarme qu'elle impute à ses sœurs, la jeune femme descend à nouveau mais, contre toute attente, elle trouve le manoir vide. Soudain, elle se retrouve plaquée contre un mur par ce qui paraît être un fantôme, avant de se voir déshabillée puis caressée sensuellement. Une lampe tombe. Piper et Prue sont alertées. Que s'est-il donc passé ? Les sœurs découvrent vite que cette présence invisible est celle d'Anton, démon capable de changer d'apparence. Ce dernier était en fait l'amant de la diabolique P. Russell, sorcière ayant vécu au manoir Halliwell au cours des années 1920 avec ses cousines, P. Bowen et P. Baxter (les prénoms ne sont jamais révélés mais on peut facilement les deviner). On découvre très vite que ces trois cousines sont en fait les vies antérieures des sœurs Halliwell. La résurgence du passé signifie la mort imminente de Phoebe Halliwell, de même que son destin semble intimement lié à celui de sa vie antérieure ${ }^{18}$. Le manoir exerce donc une influence néfaste puisqu'il arrime la série à un passé familial tragique et l'attache à un destin voué à se répéter, malédiction qui semble ne pas pouvoir être rompue. Mais l'héritage est déjoué et Phoebe parvient à se détacher de sa vie antérieure, à s'affranchir de l'influence mortifère du passé.

Par ailleurs, la résurgence des carrières artistiques des acteurs attache la série à un passé extradiégétique et semble hanter la série. Nous nous concentrerons essentiellement sur la filmographie d'Alyssa Milano, qui offre une belle illustration de cet argument. En 1989, les studios Disney sortent The Little Mermaid, inspiré du conte du danois Hans Christian Andersen paru en 1837. Le film d'animation suit les aventures et la jeune et très belle sirène Ariel qui rêve d'avoir des jambes pour pouvoir vivre sur la terre avec les humains et notamment Eric, prince dont elle s'est éprise. Pour donner corps et âme au personnage d'Ariel, les dessinateurs décident de s'inspirer de jeunes actrices dans le vent dont Alyssa Milano, alors vedette de la sitcom Who's the Boss ? (ABC, 1984-1992). À partir de photos de l'actrice et en s'inspirant de sa personnalité, ils ont ainsi façonné l'apparence et le caractère de l'héroïne de leur dessin animé. C'est en gardant à l'esprit cette anecdote biographique que l'on doit aborder l'épisode «A Witch's Tail » (5.1-2) qui ouvre la saison 5. En voulant aider Mylie, une sirène devenue humaine, qui rappelle étrangement Ariel, Phoebe se métamorphose en sirène (voir figure 5). L'influence du dessin animé est ici à l'œuvre et la référence évidente. À nouveau, la transformation se passe au manoir. Alyssa Milano, à travers le personnage de Phoebe, réincarne Ariel et on ne manquera pas de noter la ressemblance des

${ }^{18}$ L'épisode se déroule le 17 février 2000, soit 76 ans jour pour jour après la mort de P. Russell. 
costumes. Mais, alors qu'Ariel souhaite devenir humaine, Phoebe aspire davantage à la liberté que représente l'océan ; renversement qui semble indiquer un retour au passé. Néanmoins, la fin de l'épisode marque une nouvelle fois la puissance du présent face au passé. L’influence menaçante est mise en échec.

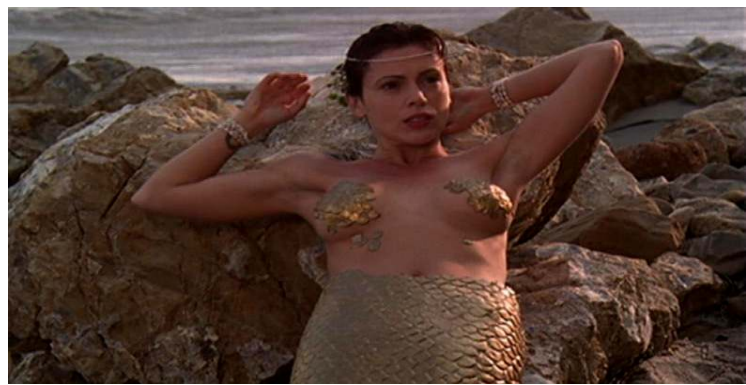

Fig. 5 : Après avoir inspiré Ariel, Alyssa Milano redevient sirène dans Charmed (5.1-2).

Ainsi, on peut arguer que tout le parcours de la série vise à se défaire de cette influence. Il semble d'ailleurs qu'un lien métaphorique se tisse entre les démons et l'intertexte, lien parfaitement mis en évidence par celui que l'on nomme «The Source». Ce démon, qui apparaît pour la première fois dans l'épilogue de la saison 3 avant d'être vaincu quelques épisodes plus tard, se révèle être l'instigateur de toutes les attaques dont ont souffert les Halliwell. Son nom, "The Source », peut être interprété de plusieurs manières : il s'agit aussi bien de la source du mal que de la source textuelle, origine de l'influence. Le manoir se serait donc imprégné de toute cette énergie démoniaque et textuelle accumulée au fil des années. D’où le recours à un "witch doctor » dans l'épisode " House Call » (5.13) pour purifier le manoir. Les sœurs Halliwell sont en effet excédées par les multiples esprits démoniaques qui hantent le manoir et font de leur vie un véritable enfer. Leo essaie tant bien que mal de rassurer Piper :

[It's] residual energy left over the years from all the demons you vanquished here. Besides the Elders said they would dissipate over time.

Cette «énergie résiduelle " condamnée à se dissiper selon l'être de lumière des sœurs ne serait-elle pas l'intertextualité " démoniaque " qui hante la série depuis ses origines ? Les Halliwell font donc appel à un guérisseur afin que ce dernier éradique tous les esprits errants. «These walls are clogged with evil waste » s'exclame-t-il après un premier repérage. L'intertexte semble présent dans chaque mur. Ce constat peut se lire comme un commentaire métafictionnel qui arrive 
juste après le centième épisode de la série. Charmed semble consciente de l'ensorcellement qui l'accable et cherche maintenant à l'éradiquer. La manière dont la série va procéder se révèle assez radicale. Envoûtée par le guérisseur, qui est convaincu que les Halliwell ne sont plus aptes à contrôler leurs obsessions et donc leurs pouvoirs, Piper va jusqu'à faire disparaître le manoir. En une formule, elle balaie ainsi tout le passé aussi bien démoniaque que textuel. Avec la disparition du manoir, la série se libère de l'angoisse bloomienne de l'influence. Désormais, elle va pouvoir affirmer son identité propre sans peur de voir surgir de nouveau l'intertexte démoniaque. C'est ce que confirme Piper dans une réplique hautement métafictionnelle :

Paige. Piper, what did you do with the house?

Piper. Oh, I thought it would be better to start from scratch.

Repartir de zéro, faire table rase du passé, voilà ce qu'annonce désormais la série. Néanmoins, Piper s'aperçoit rapidement qu'en faisant disparaitre le manoir, elle a aussi fait disparaître Phoebe. Elle recrée alors le manoir. Cette scène semble indiquer finalement qu'éradiquer l'influence serait comme détruire une partie de l'identité de la série, puisque cela revient à tuer Phoebe. La série doit alors trouver un moyen de concilier influence et originalité et non pas seulement de chercher à annihiler le charme. C'est là l'argument défendu par Markus Reisenleitner pour qui le manoir Halliwell ne représente pas tant une menace - le topos du manoir hanté fut repris par nombre d'écrivains romantiques et victoriens ${ }^{19}-$, que la source d'un immense pouvoir pour les sœurs. Cette source s'acquière par un dialogue avec le passé et non par sa négation. La connaissance du passé (l'histoire du manoir et celle de la lignée Halliwell) amène le pouvoir présent et futur :

(...) The sisters' commitment to their home and their willingness (and ability) to encounter its horrors, to bring the demons home, as it were, as well as to learn from the histories its walls remember, are presented as sources of empowerment.

Pourtant, elle réitère sa tentative, de manière plus radicale encore, dans le pénultième épisode intitulé « Kill Billie: Vol. 2 » (8.21). L'épilogue voit le combat final entre les sœurs Halliwell et les sœurs Jenkins qui, à forces égales, s'engagent dans un affrontement voué à la perte des deux parties. Alors que les déflagrations jaillissent de toutes

19 On peut penser à de nombreux romans tels Jane Eyre de l'Anglaise Charlotte Brontë (1847) ou encore The House of the Seven Gables (1851) de l'Américain Nathaniel Hawthorne dans lesquels les manoirs sont tout aussi effrayants que dangereux. On notera que, dans le roman d'Hawthorne, l'un des personnages principaux se prénomme Phoebe... Dans la même veine horrifique, "The Fall of the House of Husher » (1839), nouvelle ténébreuse écrite par Edgar Allan Poe, présente un huis-clos obsédant dans un manoir hanté. Dans toute cette littérature gothique, la menace s'incarne dans chaque mur et est le plus souvent une résurgence d'un passé douloureux et caché. 
parts, tous les objets symboliques du manoir et de la série sont détruits dans un ordre révélateur. Un premier jet percute le lustre mythique que l'on voit trembler et scintiller à plusieurs reprises au cours de la série, notamment lors de la naissance et de la renaissance du pouvoir des trois (1.01; 4.01), moments charnières qui emblématisent l'héritage et la passation de pouvoir. Puis, c'est au tour de l'horloge, détruite une vingtaine de fois au cours des huit saisons, de recevoir une charge électrique létale. Tout cycle, passé et à venir, est donc réduit en cendres. Renier le passé implique l'impossibilité d'un futur. Quelques plans plus tard, le grenier est pris par la déflagration grandissante, dernière étape avant l'explosion du manoir. Tout s'achève à l'endroit où tout a commencé. Toutes les parties symboliques de la série sont détruites ; du manoir, il ne restera qu'un champ de ruines (voir figure 6).

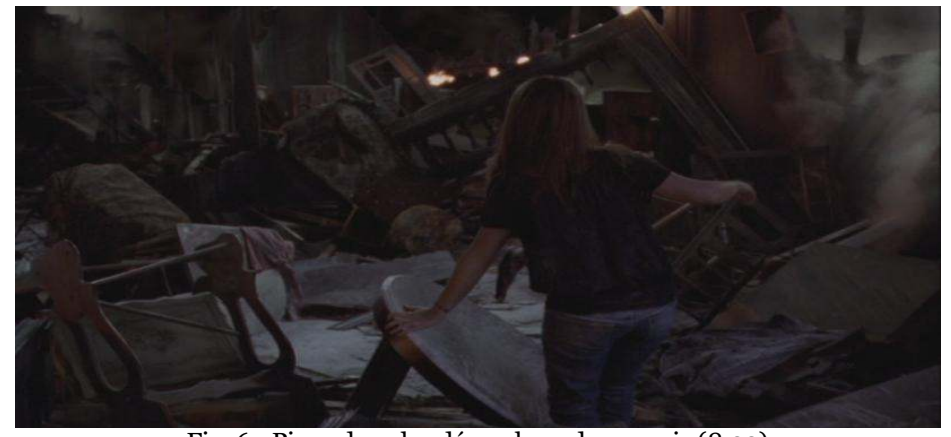

Fig. 6 : Piper dans les décombres du manoir (8.22)

En une trentaine de secondes, tout ce qui était constitutif de Charmed, jusqu'à ses héroïnes, est réduit à néant. La scène post apocalyptique qui suit montre que seule Piper a survécu. Encore une fois, la morale est univoque : éradiquer l'influence ne prélude d'aucun avenir possible. Sans origine, pas d'originalité. Et c'est finalement cette maxime qu'embrasse la série lors des dernières minutes du bien nommé "Forever Charmed » (8.22). Après avoir remporté leur ultime bataille, les sœurs Halliwell se réunissent autour du Livre des Ombres, prêtes à y inscrire leur histoire et à s'intégrer à leur tour dans le cycle éternel de l'héritage et de la transmission :

Piper. Well I think we should write everything down, everything

that happened, everything we want the futuregenerations to know so that we can pass it down just like it was passed down to us.

Cette idée est développée dans la dernière scène de l'épisode où la voix de Piper se dédouble, faisant le lien entre présent et futur. C'est en effet 
une Piper âgée de 80 ans que l'on retrouve assise avec son arrièrepetite-fille à feuilleter le Livre des Ombres. Elle lit les mots qu'elle y a inscrits quelques décennies plus tôt :

Piper. And although we certainly had our struggles and heart aches over the years we're a family of survivors and we will always be. Which is why we've truly been Charmed. Son arrière-petite-fille. Again, Grandmama, again.

Piper. Oh, dear. No, I can't. I need to rest. But you can look at it for a little while if you'd like. After all, it'll be yours one day.

"Après tout, ce livre sera tien un jour », promesse d'éternité de Charmed à son public qui peut aussi se lire comme l'héritage qu'elle s'apprête à léguer aux séries à venir. Comme le suggère le titre de l'épisode, Charmed survivra dans le cœur des fans et dans d'autres univers sériels. Après avoir été imprégnée par toutes les œuvres qui l'ont précédée, la série s'apprête à devenir le modèle pour les futures créations et à faire l'objet de tous les recyclages. Comme le résume Tiphaine Samoyault, "le rapport au modèle implique aussi une recherche de constitution de soi en modèle ${ }^{20}$. " La preuve en est avec la récente et éphémère The Secret Circle (The CW, 2011-2012), série dramatique fantastique dont beaucoup de spectateurs ont noté la filiation avec Charmed. Outre des similitudes d'ordre narratif, on peut relever notamment la présence d'un Livre des Ombres, rappel symbolique imparable du grimoire des sœurs Halliwell, ou bien une forte ressemblance architecturale - et décorative - entre la maison de l'héroïne, Cassie, et le manoir de Charmed qu'une internaute n'a pas manqué de repérer :

If you're a diehard Charmed fan who has seen the new CW show, The Secret Circle, you've probably noticed the eerie resemblance between the home of Cassie Blake and the Halliwell Manor. Other than a moved staircase, the floor plan is almost $100 \%$ identical, complete with the stained glass door ${ }^{21}$.

Charmed se présente ainsi comme une série postmoderne à bien des égards. Par son recyclage de la culture populaire et lettrée, ses réécritures multiples allant jusqu'à transgresser les niveaux narratifs, elle s'est façonnée une identité hybride et ludique parfaitement ancrée dans son époque. Cette filiation consciente et recherchée dans un premier temps s'est ensuite transformée en héritage pesant que la série a cherché à estomper. L'influence fondatrice est alors devenue indésirable - étape nécessaire dans l'affirmation d'une identité propre. Elle n'a cependant pas cessé de surgir et est peu à peu devenue un

${ }^{20}$ Tiphaine Samoyault, L'Intertextualité : Mémoire de la littérature, Paris, Armand Colin, collection 128,2005

${ }^{21} \mathrm{http}$ ://answers.yahoo.com/question/index?qid=20120217105605AA8WVle,

lien consulté le 11 décembre 2014 . 
ennemi à éliminer, incarné par le manoir Halliwell où demeurent les fantômes familiaux et culturels du passé. Nous l'avons vu, la série essaie dans ses dernières saisons de se soustraire à toutes ces influences. Mais, à faire table rase de ses origines, il semble qu'elle efface aussi une partie de ce qui la définit et fait son succès. La résolution de ce conflit entre passé et présent ne peut donc être le produit d'une négation mais plutôt d'un dialogue. Écrire son histoire ne peut se faire sans prendre en compte celle qui nous précède et c'est en embrassant ses origines et en s'inscrivant dans le cycle immuable de l'héritage et de la transmission que Charmed transcende ses ambitions premières et parvient à affirmer son originalité. Elle se situe dès lors à la croisée des chemins, entre passé, présent et futur. Le charme a opéré, opère et opèrera toujours et nous serons « Forever Charmed ».

\section{Bibliographie}

BEeler Karen \& BEELER, Stan (éds.), Investigating Charmed: The Magic Power of TV, London, I.B. Tauris, 2007.

BLoom Harold, The Anxiety of Influence: A Theory of Poetics, New York, Oxford University Press, 1997.

CRUSIE Jennifer, Totally Charmed: Demons, Whitelighters And the Power of Three, Dallas, BenBella, 2005.

GenetTe Gérard, Palimpsestes : la littérature au second degré, Paris, Éditions du Seuil, 1982.

--, Métalepse, Paris, Éditions du Seuil, 2004.

MARSHALl Jill and WERNDLY Angela, The Language of Television, London, Routledge, 2002.

O’DAY Marc, « Postmodernism and Television », in Stuart Sim (ed.), The Routledge Companion to Postmodernism, New York, Routledge, Second edition, 2005 .

Oтт Brian L., The Small Screen: How Television Equips Us to Live in the Information Age, London, Wiley-Blackwell, 2006.

Pichard Alexis, Le Nouvel âge d'or des séries américaines, Paris, Le Manuscrit, 2011.

SAmoyault Thiphaine, L'Intertextualité : Mémoire de la littérature, Paris, Armand Colin, 2005. 


\section{L'auteur}

Alexis Pichard est agrégé d'anglais et ATER à l'université Paris Ouest Nanterre la Défense. Il prépare une thèse de doctorat sur les séries américaines de l'après 11-Septembre et il est notamment l'auteur du Nouvel âge des séries américaines (Le Manuscrit, 2011, $2^{\mathrm{e}}$ éd. 2013) et des Séries américaines, la société réinventée ? (L’Harmattan, 2013) avec Aurélie Blot. 